\title{
BHASKAR RAO DESIGNS FROM CYCLOTOMY
}

\author{
DEBORAH J. STREET
}

(Received 8 November 1979)

Communicated by W. D. Wallis

\begin{abstract}
A Bhaskar Rao design is obtained from the incidence matrix of a partially balanced incomplete block design with $m$ associate classes by negating some elements of the matrix in such a way that the inner product of rows $\alpha$ and $\beta$ is $c_{i}$ if $\alpha$ and $\beta$ are ith associates. In this paper we use nested designs constructed from unions of cyclotomic classes to give Bhaskar Rao designs.
\end{abstract}

1980 Mathematics subject classification (Amer. Math. Soc.) : 05 B 20, 05 B 30.

\section{Introduction}

Let $X=A-B$, where $A$ and $B$ are $v \times b(0,1)$ matrices, and the Hadamard product of $A$ and $B, A * B$, is zero. Then $X$ is a Bhaskar Rao design if

(i) $X X^{\mathrm{T}}=r I+\sum_{i=1}^{m} c_{i} B_{i}$,

(ii) $N=A+B$ satisfies $N N^{\mathrm{T}}=r I+\sum_{i=1}^{m} \lambda_{i} B_{i}$ (that is, $N$ is the incidence matrix of a $\operatorname{PBIBD}(m))$.

Such a matrix $X$ will be denoted by $\operatorname{BRD}\left(v, b, r, k ; \lambda_{1}, \ldots, \lambda_{m} ; c_{1}, \ldots, c_{m}\right)$.

These designs have been considered by several authors. They are a generalization of weighing matrices (Geramita and Seberry (1979)) and are useful in the construction of PBIBDs (Bhaskar Rao (1970); Dey and Midha (1976); Street and Rodger (1979)).

We now introduce some notation useful in the remainder of the paper. As in Morgan et al. (1976): $A \& B$ is the collection of all the elements of $A$ and $B$, preserving multiplicity; $A+B$ is the collection of nonzero sums $a+b, a \in A, b \in B$, preserving multiplicity; $A-B$ is similarly defined with nonzero differences; $n A$ is the collection of $n$ copies of $A$. As in Storer (1967), the cyclotomic number $(i, j)$ is the number of ordered pairs $s, t$ such that

$$
x^{e s+i}+1=x^{e t+j} \quad(0 \leqslant s, t \leqslant f-1),
$$


where $x$ is a primitive root of $\mathrm{GF}\left(p^{n}\right), p^{n}=e f+1$. If there is doubt as to which factorization of $p^{n}-1$ is being used, it is specified by writing $(i, j)_{e}$.

Let $G$ be an abelian group with its elements ordered as $g_{1}, g_{2}, \ldots, g_{v}$ in some way. Let $T$ be a subset of $G$ and suppose $T=R \cup S$, where $R \cap S=\phi$. We will denote by $(R,+: S,-)$ the matrix $A=\left(a_{i j}\right)$ with

$$
a_{i j}=\left\{\begin{aligned}
1 & g_{j}-g_{i} \in R, \\
-1 & g_{j}-g_{i} \in S, \\
0 & \text { otherwise. }
\end{aligned}\right.
$$

The matrix obtained by squaring each element of $A$ is the usual incidence matrix of $T$.

The following lemma appears in Street and Rodger (1979) for the case $m=1$; the proof is analogous.

Lemma 1. Let $T_{1}, T_{2}, \ldots, T_{n}$ be the initial blocks of a $\operatorname{PBIBD}(m)$ and assume the elements of the ith associate class occur $\lambda_{i}$ times. Thus

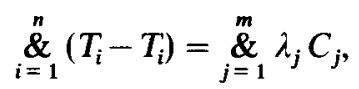

where $C_{j}$ is the jth associate class. Suppose $T_{i}=R_{i} \cup S_{i}, R_{i} \cap S_{i}=\emptyset, 1 \leqslant i \leqslant n$, and

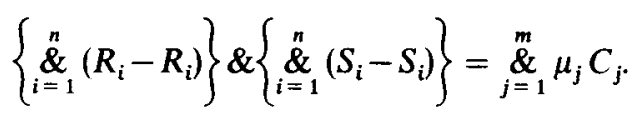

Then $X=\left[\left(R_{1},+; S_{1},-\right):\left(R_{2},+; S_{2},-\right): \ldots:\left(R_{n},+; S_{n},-\right)\right]$ is a $\operatorname{BRD}(v, n v, n k, k$; $\left.\lambda_{1}, \ldots, \lambda_{m} ; 2 \mu_{1}-\lambda_{1}, \ldots, 2 \mu_{m}-\lambda_{m}\right)$.

In the remainder of the paper we use results of Lehmer (1974), Homel and Robinson (1975) and Morgan et al. (1976) to construct sets which satisfy the conditions of this lemma.

\section{Nested block designs}

Lehmer (1974) has given a family of supplementary difference sets (sds). Her method is used to give related families of sds.

LemMa 2. Let $p^{n}=2 m f+1$ be a prime power. Let $f$ be odd and let $x$ be a primitivé root of $\mathrm{GF}\left(p^{n}\right)$. Denote the cyclotomic classes with $e=2 \mathrm{~m}$ by

$$
C_{i}=\left\{x^{2 m s+i} \mid s=0,1, \ldots, f-1\right\}, \quad i=0,1, \ldots, 2 m-1 .
$$


Let $i_{0}=0, i_{1}, \ldots, i_{m-1}$ be a complete set of residues modm such that $0 \leqslant i_{j} \leqslant 2 m-1$ for every $j$ and let $A$ be a subset of $\{0,1, \ldots, m-1\}$. Then the $m$ sets

$$
T_{h}=\bigcup_{j \in A} C_{i_{j}-i_{h}}, \quad h=0,1, \ldots, m-1
$$

are $m-\{2 m f+1 ; t f ; t(t f-1) / 2\}$ sds, where $t=|A|$.

Proof. We see that

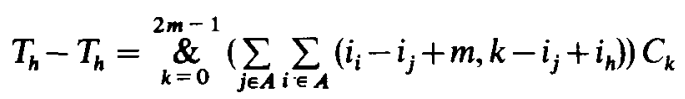

and

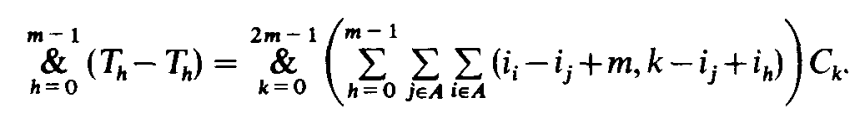

As $f$ is odd,

$$
\left(i_{i}-i_{j}+m, k-i_{j}+i_{h}\right)=\left(i_{j}-i_{i}+m, k+m+i_{h}-i_{i}\right)
$$

and

$$
\sum_{h=0}^{m-1}\left(i_{i}-i_{j}+m, k-i_{j}+i_{h}\right)+\sum_{h=0}^{m-1}\left(i_{i}-i_{j}+m, k+m+i_{h}-i_{j}\right)=f-\delta_{i j},
$$

where $\delta_{i j}$ is the Kronecker delta. Summing over both $i$ and $j$, we obtain

$$
\begin{aligned}
\lambda & =\sum_{j \in A} \sum_{i \in A} \sum_{h=0}^{m-1}\left(i_{i}-i_{j}+m, k-i_{j}+i_{h}\right) \\
& =\sum_{j \in A} \sum_{i \in A} \sum_{h=0}^{m-1}\left(i_{j}-i_{i}+m, k+m+i_{h}-i_{i}\right) \\
& =t(t f-1) / 2, \quad \text { as required. }
\end{aligned}
$$

THEOREM 1. Let $v=p^{n}=2 m f+1$ be a prime power, where $f$ is odd. Then there exists $a$

$$
\operatorname{BRD}\left(v, m v, t f, m t f ; t(t f-1) / 2 ;\left(4 r^{2}-4 t f r+t^{2} f-t\right) / 2\right)
$$

for all $1 \leqslant r<t \leqslant m$.

ProOf. Let $A$ be a subset of $\{0,1, \ldots, m-1\}$ of order $t$ such that $A=B \cup C$, where $B \cap C=\emptyset$ and $B$ is of order $r$. Then let

$$
T_{h}=\bigcup_{j \in A} C_{i_{j}-i_{h}}, \quad R_{h}=\bigcup_{j \in B} C_{i_{j}-i_{h}} \text { and } S_{h}=\bigcup_{j \in C} C_{i_{j}-i_{h}}
$$

(where $i_{j}$ and $C_{i}$ are as defined in Lemma 2); the result follows from Lemmas 1 and 2. 
COROLLARY 1. Let $v=p^{n}=2 m f+1$ be a prime power, where $f$ is odd. Then there exists $a \operatorname{BRD}\left(v, m v, a^{2} f^{2}, m a^{2} f^{2} ; a^{2} f\left(a^{2} f^{2}-1\right) / 2 ; 0\right)$ for all $0<a^{2} f \leqslant m$.

COROLlary 2. Let $v=p^{n}=2 m f+1$ be a prime power, where $f$ is odd. Then there exists a $\operatorname{BRD}(v, m v, 2 n f, 2 m n f ; n(2 n f-1) ;-n)$ for all $0<2 n \leqslant m$.

\section{Nested PBIBDs}

In this section we use the notation of Morgan et al. (1976); in addition let

$$
M_{i}=\left\{x^{a 2 \alpha \beta+i} \mid a=0,1, \ldots, \gamma-1\right\}, \quad i=0,1, \ldots, 2 \alpha \beta-1,
$$

where $x$ is a primitive root of GF $\left(p^{n}\right)$ and $p^{n}=2 \alpha \beta \gamma+1$, where $p$ is an odd prime and $n, \alpha, \beta, \gamma$ are positive integers with $\alpha, \beta, \gamma \geqslant 2$.

Choose an integer $t$ such that $0<t \leqslant 2 \alpha \beta$, and $t$ distinct integers $a_{1}, a_{2}, \ldots, a_{t}$ such that $0 \leqslant a_{1}<a_{2}<\ldots<a_{t} \leqslant 2 \alpha \beta-1$. Define

$$
G_{i}=\bigcup_{i=1}^{t} M_{a_{h}+i}, \quad i=0,1, \ldots, 2 \alpha \beta-1
$$

and

$$
H_{i}=\{0\} \cup G_{i}, \quad i=0,1, \ldots, 2 \alpha \beta-1
$$

LEMMA 3. If $\gamma$ is odd, $-M_{i}=M_{i+\alpha \beta}$ and

$$
G_{i}-G_{i}=\underset{k=0}{2 \alpha \beta-1} g_{k} M_{k+i}, \quad i=0,1, \ldots, 2 \alpha \beta-1,
$$

where

where

$$
\begin{aligned}
g_{k}=\sum_{h=1}^{t} \sum_{j=1}^{t}\left(a_{j}-a_{h}+\alpha \beta, k-a_{h}\right)_{2 \alpha \beta}, \\
H_{i}-H_{i}={ }_{k=0}^{2 \alpha \beta-1} h_{k} M_{k+i}, \quad i=0,1, \ldots, 2 \alpha \beta-1
\end{aligned}
$$

$$
h_{k}=g_{k}+\sum_{j=1}^{t} \delta_{a_{j}, k}+\sum_{j=1}^{t} \delta_{a_{j}+\alpha \beta, k}
$$

The designs constructed in the following theorem generalize Theorem 2.7 of Homel (1972) (see also Homel and Robinson (1975)).

THEOREM 2. Let $\beta \gamma$ be odd, and let $p^{n}=2 \alpha \beta \gamma+1$ be a prime power. Then the $\beta$ sets $G_{2 a j}, j=0,1, \ldots, \beta-1$, or the $\beta$ sets $H_{2 \alpha j}, j=0,1, \ldots, \beta-1$, may be used as the initial 
blocks of $a \operatorname{PBIBD}(m)$ where $v=p^{n}, b=\beta v, m \leqslant \alpha$ and each associate class consists of a cyclotomic class

$$
C_{k}=\left\{x^{a \alpha+k} \mid a=0,1, \ldots, 2 \beta \gamma-1\right\} \quad k=0,1, \ldots, \alpha-1
$$

or a union of such classes. Table 1 contains the parameters of the design when $m=\alpha$.

TABLE 1.

\begin{tabular}{lcccccc}
\hline \multicolumn{1}{c}{$\begin{array}{c}\text { Initial } \\
\text { blocks }\end{array}$} & $k$ & & \multicolumn{4}{c}{ Parameters when $m=\alpha$} \\
\hline (i) $G_{2 \alpha j}$ & $t \gamma$ & $\beta k$ & $2 \beta \gamma$ & $\sum_{i}$ & $\sum_{i}$ & $p_{i j}^{k-1} g_{i+2 \alpha j}$ \\
(ii) $H_{2 \alpha j}$ & $t \gamma+1$ & $\beta k$ & $2 \beta \gamma$ & $\sum_{j=0}^{\substack{j=0 \\
\beta-1}} h_{i+2 \alpha j}$ & $(k-j, i-j)_{\alpha}$ \\
\hline
\end{tabular}

ProOf. (i) From Lemma 3 we have

$$
\begin{aligned}
& \underset{j=0}{\beta-1}\left(G_{2 \alpha j}-G_{2 \alpha j}\right)=\underset{k=0}{2 \alpha \beta-1} g_{k} \underset{j=0}{\beta-1} M_{2 \alpha j+k} \\
& =\sum_{k=0}^{2 \alpha-1}\left(\sum_{i=0}^{\beta-1} g_{k+2 \alpha i}\right) C_{k}^{(2 \alpha)},
\end{aligned}
$$

where $C_{k}^{(2 \alpha)}$ is the $k$ th cyclotomic class with $e=2 \alpha$. Now

and

$$
C_{k}=C_{k}^{(2 \alpha)} \cup C_{k+\alpha}^{(2 \alpha)}
$$

$$
\begin{aligned}
\sum_{i=0}^{\beta-1} g_{k+2 \alpha i} & =\sum_{i=0}^{\beta-1}\left(\sum_{h=1}^{t} \sum_{j=1}^{t}\left(a_{j}-a_{h}+\alpha \beta, k+2 \alpha i-a_{h}\right)_{2 \alpha \beta}\right) \\
& =\sum_{i=0}^{\beta-1}\left(\sum_{h=1}^{t} \sum_{j=1}^{t}\left(a_{h}-a_{j}+\alpha \beta, k+\alpha(2 i+\beta)-a_{j}\right)_{2 \alpha \beta}\right) \\
& =\sum_{i=0}^{\beta-1} g_{k+\alpha(2 i+1)}
\end{aligned}
$$

if $\beta$ is odd. Thus the associate classes are as claimed. As in Morgan et al. (1976), $p_{i j}^{k}=p_{j i}^{k}$ as $2 \beta \gamma$ is even.

The proof of (ii) is similar.

Let $A$ and $B$ be two disjoint sets of $t$ and $s$ distinct integers between 0 and $\alpha \beta-1$. Let $C=A \cup B$ and let $E_{j \alpha}^{\prime}, E_{j \alpha}^{\prime \prime}$, and $E_{j \alpha}, 0 \leqslant j \leqslant \beta-1$, be the initial blocks for the construction of Morgan et al. (1976), Theorem 2, using the sets $A, B$ and $C$ respectively (thus $\beta \gamma$ is even). Let the parameters of these designs be $n_{i}, \lambda_{i}, p_{i j}^{k}$; $n_{i}, \mu_{i}, p_{i j}^{k}$; and $n_{i}, v_{i}, p_{i j}^{k}$ respectively and note that $E_{j \alpha}=E_{j \alpha}^{\prime} \cup E_{j \alpha}^{\prime \prime}$. Similarly let $F_{j \alpha}^{\prime}=E_{j \alpha}^{\prime} \cup\{0\}, F_{j \alpha}^{\prime \prime}=E_{j \alpha}^{\prime \prime} \cup\{0\}$ and $F_{j \alpha}=E_{j \alpha} \cup\{0\}$ (so $F_{j \alpha}=E_{j \alpha}^{\prime} \cup F_{j \alpha}^{\prime \prime}$ ), with parameters $n_{i}, \lambda_{i}^{\prime}, p_{i j}^{k} ; n_{i}, \mu_{i}^{\prime}, p_{i j}^{k}$; and $n_{i}, v_{i}^{\prime}, p_{i j}^{k}$ respectively. 
THEOREM 3. Let $v=p^{n}=\alpha \beta \gamma+1$ be a prime power, where $\beta \gamma$ is even. Then there exist:

(i) $\operatorname{BRD}\left(v, \beta v, \beta k,(t+s) \gamma ; v_{1}, \ldots, v_{\alpha} ; 2\left(\lambda_{1}+\mu_{1}\right)-v_{1}, \ldots, 2\left(\lambda_{\alpha}+\mu_{\alpha}\right)-v_{\alpha}\right)$;

(ii) $\operatorname{BRD}\left(v, \beta v, \beta k,(t+s) \gamma+1 ; v_{1}^{\prime}, \ldots, v_{\alpha}^{\prime} ; 2\left(\lambda_{1}^{\prime}+\mu_{1}^{\prime}\right)-v_{1}^{\prime}, \ldots, 2\left(\lambda_{\alpha}^{\prime}+\mu_{\alpha}^{\prime}\right)-v_{\alpha}^{\prime}\right)$.

ProOf. Apply Lemma 1 to the designs discussed above.

We may use the designs of Theorem 2 in a similar construction; here

$$
\begin{aligned}
& v_{i}=\sum_{j=0}^{\beta-1}\left(\sum_{x \in C} \sum_{y \in C}(y-x+\alpha \beta, i+2 \alpha j-x)_{2 \alpha \beta}\right), \\
& v_{i}^{\prime}=v_{i}+\sum_{j=0}^{\beta-1}\left(\sum_{x \in C}\left(\delta_{x, i+2 \alpha j}+\delta_{x+\alpha \beta, i+2 \alpha j}\right)\right) \text { and so on. }
\end{aligned}
$$

THEOREM 4. Let $v=p^{n}=2 \alpha \beta \gamma+1$ be a prime power, where $\beta \gamma$ is odd. Then there exist:

(i) $\operatorname{BRD}\left(v, \beta v, \beta k,(t+s) \gamma ; v_{1}, \ldots, v_{\alpha} ; 2\left(\lambda_{1}+\mu_{1}\right)-v_{1}, \ldots, 2\left(\lambda_{\alpha}+\mu_{\alpha}\right)-v_{\alpha}\right)$;

(ii) $\operatorname{BRD}\left(v, \beta v, \beta k,(t+s) \gamma+1 ; v_{1}^{\prime}, \ldots, v_{\alpha}^{\prime} ; 2\left(\lambda_{1}^{\prime}+\mu_{1}^{\prime}\right)-v_{1}^{\prime}, \ldots, 2\left(\lambda_{\alpha}^{\prime}+\mu_{\alpha}^{\prime}\right)-v_{\alpha}^{\prime}\right)$.

Homel and Robinson (1975) give a number of nested designs which may also be used in a similar construction.

\section{References}

M. Bhaskar Rao (1970), 'Balanced orthogonal designs and their applications in the construction of some BIB and group divisible designs', Sankhyā Ser. A 32, 439-448.

A. Dey and C. K. Midha (1976), 'Generalized balanced matrices and their applications', Utilitas Math. 10, 139-149.

Anthony V. Geramita and Jennifer Seberry (1979), Orthogonal designs: quadratic forms and Hadamard matrices (Marcel Dekker, New York and Basel).

R. J. Homel (1972), The construction and analysis of partially balanced incomplete block designs (M.Sc. thesis, University of Sydney).

R. J. Homel and J. Robinson (1975), 'Nested partially balanced incomplete block designs', Sankhyā Ser. B 37, 201-210.

Emma Lehmer (1974), 'A family of supplementary difference sets', Bull. Austral. Math. Soc. 11, 1-4.

Elizabeth J. Morgan, Anne Penfold Street and Jennifer Seberry Wallis (1976), 'Designs from cyclotomy', Combinatorial Math. IV, Lecture Notes in Mathematics 560 (editors Louis R. A. Casse and Walter D. Wallis), pp. 158-176 (Springer-Verlag, Berlin, Heidelberg, New York).

Thomás Storer (1967), Cyclotomy and difference sets (Markham, Chicago).

Deborah J. Street and Christopher A. Rodger (1979) 'Some results on Bhaskar Rao designs' (preprint).

Department of Mathematical Statistics

University of Sydney

N.S.W. 2006

Australia 